Produto \& Produção, vol. 19, n.1, p.19-33. 2018*

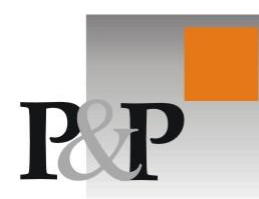

\author{
Valdir Silveira Júnior \\ Faculdade Farias Brito (FFB) \\ valdirsjr@gmail.com \\ Maurício Johnny Loos \\ Universidade Federal de Santas Catarina (UFSC) \\ mauricioloos@hotmail.com
}

\title{
Aplicação da Prototipagem Rápida no desenvolvimento de novos productos: um estudo de caso em uma empresa de tecnología do estado do Ceará
}

\section{Resumo}

A área de Pesquisa e Desenvolvimento de novos produtos, em especial eletrônicos, tem crescido consideravelmente, ganhando espaço em um cenário no qual a inovação passa a ser um fator decisivo. Contudo, o desenvolvimento de produtos inovadores requer alto investimento e capacidade técnica a fim de atender as demandas e requisitos, ao mesmo tempo com o desafio de buscar maior sintonia com o mercado. É neste contexto que surge a Prototipagem Rápida (PR) como uma solução tecnológica para o desenvolvimento mais eficaz de protótipos, contribuindo para a construção de um produto final com mais qualidade e tempo de resposta adequado à exigência do mercado. $O$ presente artigo investiga, por meio de um Estudo de Caso, a aplicação das tecnologias de Prototipagem Rápida em uma empresa de hardware e o impacto no tempo e custo no desenvolvimento de protótipos. Os resultados mostram que o uso da prototipagem rápida possibilitou melhorias significativas no processo de desenvolvimento da empresa, contribuindo para a redução de desperdícios e maior agilidade do projeto até a o produto final.

Palavras-chave: Desenvolvimento de Produtos, Prototipagem Rápida

\begin{abstract}
The area of Research and Development of new products, especially electronic products, has grown considerably, gaining space in a scenario in which innovation becomes a decisive factor. However, the development of innovative products requires high investment and technical capacity to meet the demands and requirements, at the same time with the challenge of seeking greater market alignment. It is in this context that Rapid Prototyping emerges as a technological solution for the most efficient development of prototypes that contribute to the construction of a product with higher quality and response time adequate to the market demand. The present article investigates, through a Case Study, the application of Rapid Prototyping technologies in a hardware company and the impact of these technologies on time and cost in the development of prototypes. The results show that the use of rapid prototyping has resulted in significant improvements for the development process, contributing to the waste reduction and greater agility from the project to the final product.
\end{abstract}

Keywords: Product Development, Rapid Prototyping 


\section{Introdução}

O desenvolvimento de um novo produto compreende um processo, com atividades e fases, desde a modelagem da ideia até a entrega de todas as informações necessárias para que a produção seja capaz de fabricar o produto, onde busca-se atender a necessidade do mercado, considerando as restrições tecnológicas e estratégicas competitivas (ROZENFELD et al., 2006).

Uma importante etapa desse processo é a concepção de um protótipo, que pode ser conceituado como um modelo ou materialização da ideia ou versão preliminar de um produto, para que possa ser testado e validado antes da produção definitiva (KAMINSKI et al., 2015).

Aliado a esses aspectos, atualmente temos um mercado consumidor exigente e empresas mais competitivas. Estes fatores repercutem em uma maior "urgência" para o lançamento de novos produtos no mercado, e ao mesmo tempo produtos que atendam as diversas camadas de necessidades e requisitos. Esse cenário motiva a busca das empresas pela redução do ciclo de produção e uma melhor e mais rápida concepção dos seus produtos, sendo a criação de protótipos uma etapa importante no desenvolvimento de um novo produto (ELEVERUM et al., 2015). Neste sentido, o investimento, tempo e esforço necessários para conceber um novo produto aumentam consideravelmente, forçando as empresas a buscarem ferramentas e técnicas para melhorar esse processo.

O conjunto de tecnologias denominada Prototipagem Rápida, (PR) ou Rapid Prototyping, é uma maneira inovadora e cada vez mais em evidência como uma solução acessível para construção dos protótipos. Sua utilização contribui para a redução de erros, tempo e custos relacionados ao projeto, permitindo que, em um curto espaço de tempo, seja possível validar conceitos, soluções, adequação e viabilidade relacionadas ao produto (VOLPATO, 2007).

Diante disso, torna-se relevante investigar a utilização desse conjunto de tecnologias como um fator de inovação dentro das empresas que atuam com Pesquisa, Desenvolvimento e Inovação (PD\&I), a exemplo da empresa deste Estudo de Caso, e como objetivo de pesquisa observar e analisar o impacto da Prototipagem Rápida no ciclo de desenvolvimento de novos produtos, enfatizando o caso da produção de dispositivos eletrônicos.

$\mathrm{O}$ presente artigo, neste sentido, primeiramente estabelece o referencial teórico, seguido pelos procedimentos metodológicos adotados, resultados empíricos e, finalmente, suas conclusões.

\section{Referencial Teórico}

A seguir é apresentada uma fundamentação teórica referente ao Desenvolvimento de Produtos e Prototipagem Rápida, no intuito de estabelecer a visão da literatura para, posteriormente, poder-se efetuar as análises de confronto com o trabalho de campo observado no objeto de análise.

\subsection{Desenvolvimento de Produtos}

O processo de desenvolvimento de produtos pode ser entendido como sendo um conjunto de procedimentos sistematizados através dos quais busca-se atender as necessidades dos clientes, por meio de soluções e ferramentas adequadas, que contemplem os aspectos, recomendações, imposições, limitações e restrições relacionadas aos campos de conhecimento envolvidos no processo (ROZENFELD et al. 2006).

Ainda segundo Rozenfeld et al. (2006), o processo de desenvolvimento de produtos (PDP) é um processo de negócio cada vez mais decisivo devido ao comércio internacional, competitividade entre as empresas, disponibilidade dos meios tecnológicos, aumento da variedade de produtos e a diminuição dos seus ciclos de vida, significando entrada de novos produtos que visam atender partes peculiares do mercado, aliando novas tecnologias e se adequando a novos padrões e restrições legais. O desenvolvimento de novos produtos oferece riscos para a empresa, principalmente pelos fatores da orientação do produto ao mercado, planejamento e especificações, e os fatores internos da empresa.

Para Takahashi e Takahashi (2007), existem várias decisões que o projeto precisa realizar para englobar as áreas técnicas e econômicas de mercado. $\mathrm{O}$ ajuste de decisões das fases de 
desenvolvimento busca reduzir as incertezas ao longo do tempo, definindo o processo em 5 fases: 1) Avaliação de conceito: análise das oportunidades de produto; 2) Planejamento e especificação: produto claro, conciso, com as vantagens competitivas, funções e viabilidade; 3) Desenvolvimento: realização do planejamento e detalhamento do projeto; 4) Teste e avaliação: realização de um teste final, preparação da produção e o lançamento do produto; 5) Liberação do produto: nessa fase verificase a produção, marketing, distribuição e o suporte ao produto.

Já Rozenfeld et al. (2006), definem o desenvolvimento de produtos em um modelo referencial aplicado no PDP, exposto na figura 1, representado por três macrofases: prédesenvolvimento, desenvolvimento e pós-desenvolvimento, contando com os processos de apoio: gerenciamento de mudanças de engenharia e melhoria do processo.

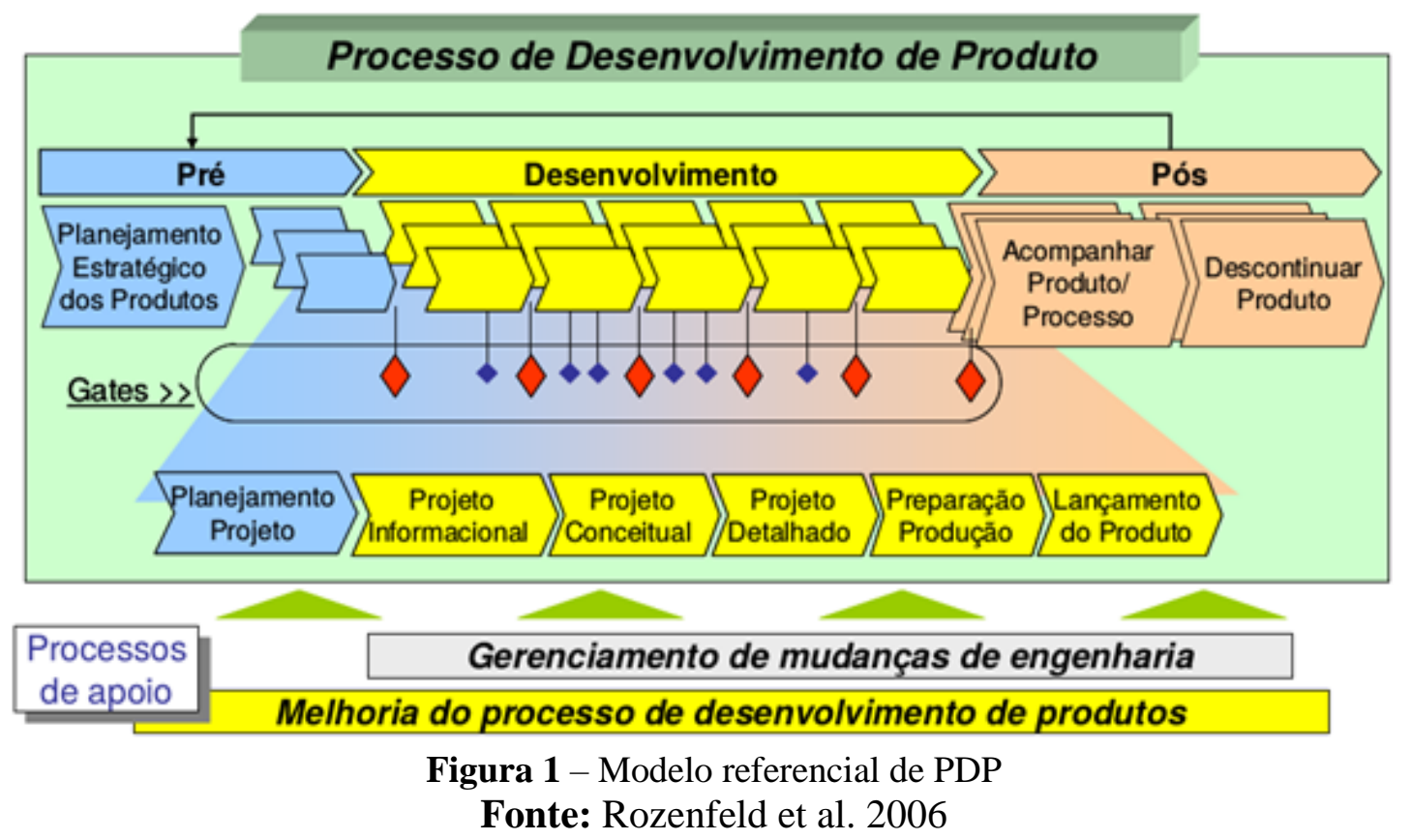

O Pré-desenvolvimento compreende as atividades para estabelecer o produto a ser desenvolvido, o escopo, avaliação econômica e de risco, indicadores para monitoramento do projeto e definição de planos de negócio. É importante compreender as necessidades e expectativas do cliente para planejar e definir o produto. Nesta fase podem ser desenvolvidas pesquisas e benchmarking. Ao final da fase são definidos os entregáveis da ideia pré-projetada e um cronograma com os marcos principais do projeto.

Desenvolvimento permite um número maior de atividades relacionadas com o projeto de um produto, sendo dividida em 04 etapas: Projeto Informacional; Projeto Conceitual; Projeto Preliminar e Projeto Detalhado.

No Pós-desenvolvimento ocorre um planejamento de como o produto será acompanhado e retirado do mercado. Como serão realizadas alterações, reparos de falhas e propor melhorias contínuas até atingir as metas impostas no PDP. Também contempla a retirada do produto do mercado e atitudes relacionadas ao descarte do material para o meio ambiente.

Segundo Rozenfeld et al. (2006), dentro das primeiras fases do PDP as soluções fundamentais e especificações do produto como: itens, tecnologias aplicadas, tipo de manufatura, são idealizadas e correspondem a cerca de $85 \%$ do seu custo final, sendo nessas fases a possibilidade de obter maior grau de incerteza sobre o produto, processo e como desempenhará no mercado.

No modelo proposto por Rozenfeld et al. (2006), a construção do protótipo na fase de desenvolvimento assume o propósito de aumentar as chances de detecção de problemas nas fases iniciais. Ainda nesta fase, a equipe multifuncional tem a oportunidade de detectar itens críticos, problemas de importação, desenvolvimento de dispositivos, entrega de protótipos, falta de capacidade de uma máquina ou equipamento de teste, sendo todos estes problemas tratados como falhas 
potenciais. Com o auxílio da ferramenta FMEA ("Failure Model and Effect Analysis" ou Análise de modo de falha e efeito) estas falhas potenciais de projeto e processo são analisadas (PINHO, 2006).

Para Barbosa (2009), o desenvolvimento de um produto para o mercado vai além da transformação de uma ideia em um projeto para fabricação, já que hoje o consumidor, com um perfil mais exigente, já observa outros aspectos, incluindo durabilidade e estética, com atenção ao design.

A preocupação com o design e a inovação é o ponto central da abordagem do modelo Design Thinking proposto por Brown (2008), que no que se refere a processos de inovação, é formado por três fases. A primeira fase é a de Inspiração, que consiste em observar e entender o problema, definindo um ponto de vista. A segunda fase é a Ideação, gerando todas as ideias possíveis e construindo protótipos para as idéias mais promissoras, testando e validando esses protótipos em ciclos de iteração antes da terceira fase, de Implementação, onde a construção da solução/produto final é realizada.

THE DESIGN THINKING PROCESS

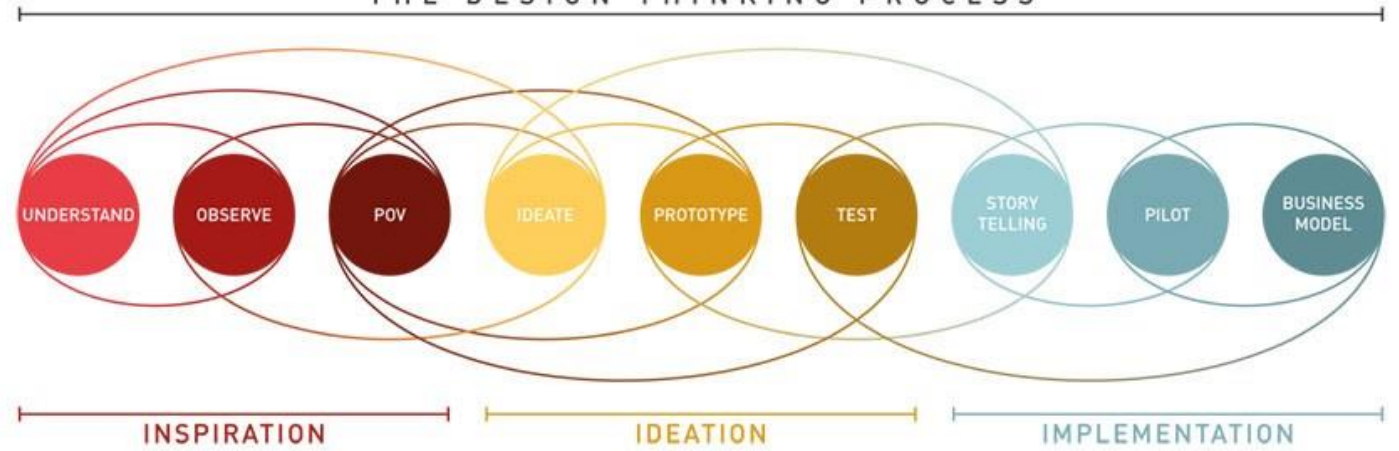

Figura 2 - Modelo Design Thinking

Fonte: www.interaction-design.org

Brown (2010, p. 83) considera a prototipagem como “[...] um dos processos mais fundamentais por meio dos quais exploramos o universo, liberamos nossa imaginação e abrimos a mente para novas possibilidades". Neste sentido, torna-se notável a construção do protótipo como uma etapa fundamental da concepção de um novo produto ou solução inovadora, permitindo a experimentação rápida, o aprendizado e a melhoria da ideia, assim como o papel da comunicação nas iterações do usuário final com o protótipo a fim de reduzir erros e garantir o sucesso da inovação.

\subsection{Prototipagem Rápida}

O termo Prototipagem Rápida refere-se a um conjunto de tecnologias utilizadas na fabricação de modelos tridimensionais, formados por camadas planas sucessivas através de informações geométricas extraídas de um sistema CAD (Computer Aided Design). Essa tecnologia é empregada por impressoras 3D, o que permite a aplicação em várias áreas do conhecimento científico e tecnológico, estando fortemente relacionada na melhoria do desenvolvimento de produtos, reduzindo tempo, custo e possibilidade de falhas. Estes fatores são determinantes na busca da competitividade de mercado melhorando a qualidade do produto (SANCHES, 2015).

A implementação de novas ferramentas e técnicas nos processos de produção é determinante, de forma que, o processo de fabricação denominada prototipagem rápida auxiliada por softwares em sistema CAD e executadas por impressoras 3D surge como um dos mais promissores processos de fabricação, sendo já utilizados nos mais variados setores da indústria.

De acordo com Gorni (2001), o termo prototipagem rápida indica um conjunto de tecnologias empregadas para a fabricação de objetos físicos de fontes de dados determinados por sistemas de projeto, amparado por computador. A prototipagem rápida é uma tecnologia inovadora 
que vem crescendo nas últimas décadas. Ela almeja confeccionar protótipos de forma relativamente rápida para verificação visual, avaliação ergonômica, análise de forma/dimensional e como padrão instrutor para a produção de ferramentas para ajudar na redução de tempo do processo de ampliação de produtos.

Para Palm (1998 apud SANCHES 2015) o termo prototipagem rápida (PR) corresponde a uma categoria de tecnologias que pode automaticamente estabelecer modelos físicos a partir de dados de um projeto. A utilização da prototipagem rápida no processo de desenvolvimento de produtos proporciona, de acordo com Modeen (2005), os consequentes pontos fortes: sua competência de produzir formas tridimensionais difíceis e detalhadas; a diminuição de lead times para peças únicas; a probabilidade de sua instalação em ambientes não industriais, que requeiram à baixa geração de ruídos ou desperdícios.

De acordo com Volpato (2007), a prototipagem rápida pode acelerar o processo de desenvolvimento de produtos, principalmente possibilitando testes a partir de modelos construídos, em curto espaço de tempo, evitando falhas futuras no produto e facilitando a comunicação entre os membros do projeto. A prototipagem rápida permite também reduzir custos e trabalhar detalhes precisos na peça com mais liberdade e agilidade do que um processo convencional e, conforme o caso, gerar a própria peça pronta para uso, sendo esse processo conhecido como Manufatura Rápida (MR).

Uma impressora 3D funciona basicamente com a construção do molde do objeto em um software virtual, logo em seguida, a impressora recebe as informações do programador, que calcula a quantidade de material necessário e o tempo estimado para ficar pronto, portanto, a impressão 3D é obtida por meio de processos de adição de determinado material na sobreposição de diversas camadas que irão dar forma ao objeto projetado virtualmente.

Impressão 3D é difundida como manufatura aditiva, onde o modelo digital 3D é salvo no formato STL (Standard Template Library), e o formato se caracteriza por ser uma forma simples e robusta de representar modelos tridimensionais através de uma lista não ordenada de triângulos irregulares. O arquivo STL possui representações numéricas para os pontos de suas coordenadas cartesianas o que possibilita que qualquer sistema de prototipagem rápida interprete seus comandos e imprima a camada de desenho por camada e formar um objeto real.

Entre os diversos sistemas de prototipagem rápida, o de Modelagem por Deposição de Material Fundido (FDM, Fused Deposition Modeling) tem se destacado por ser o mais comum encontrado nas impressoras 3D. Neste modelo, filamentos de resina termoplástica aquecida são extrudadas a partir de uma matriz em forma de ponta que se move num plano X-Y. A matriz de extrusão controlada deposita filetes de material muito finos sobre a plataforma de construção, formando a primeira camada do componente. A plataforma é mantida sob uma temperatura inferior à do material, de forma que a resina termoplástica endurece rapidamente. Após esse endurecimento a plataforma se abaixa ligeiramente e a matriz de extrusão deposita uma segunda camada sobre a primeira. O processo é repetido até a construção total do protótipo. São construídos suportes durante a fabricação para segurar o protótipo durante sua fabricação. Tais suportes são fixados ao protótipo usando-se um segundo material, mais fraco, ou uma junção perfurada. As resinas termoplásticas adequadas a esse processo incluem poliéster, polipropileno, ABS, elastômeros e cera usada no processo de fundição por cera perdida (GORNI 2001).

Os processos de prototipagem rápida atualmente existentes são constituídos por cinco etapas básicas: 1) Criação de um modelo CAD da peça que está sendo projetada; 2) Conversão do arquivo CAD em formato STL, próprio para estereolitografia; 3) Fatiamento do arquivo STL em finas camadas transversais; 4) Construção física do modelo, empilhando-se uma camada sobre a outra; 5) Limpeza e acabamento do protótipo (GORNI 2001).

Portanto, a prototipagem rápida é caracterizada por:

- Alta flexibilidade: considerando que a impressora 3D utiliza o desenho do produto digitalizado é possível mudar o produto a ser fabricado de maneira ágil, principalmente se o material utilizado se mantiver constante.

- Baixo custo unitário: a prototipagem rápida permite a produção de uma única peça com reduzido tempo de preparação de máquina, dessa forma alcançando um custo unitário baixo.

- Setup rápido: tendo em vista que os produtos a serem gerados pela impressora 3D são provenientes de arquivos digitais, então a mudança de um produto para outro é facilitada, necessitando apenas do comando de envio do novo arquivo para a impressora 3D. Essa 
rapidez pode sofrer alguma perda caso os produtos da fila requeiram materiais distintos ou cores muito específicas.

\section{Procedimentos Metodológicos}

Esta pesquisa trata-se de um estudo de caso, que Yin (2015) define como uma pesquisa empírica que analisa um fenômeno a partir da realidade, classificado como exploratório e descritivo, proporcionando maior familiaridade com o problema (GERHARDT; SILVEIRA, 2009). O estudo adota uma abordagem qualitativa, na qual são analisados os aspectos, características e problemáticas, bem como as metodologias e práticas empregadas no desenvolvimento de produtos pela empresa estudada.

Da análise qualitativa serão evidenciadas e descritas as questões referentes ao paradigma de pesquisa e desenvolvimento predominante e as soluções encontradas pela empresa no âmbito do projeto de novos produtos, especificamente no desenvolvimento de dispositivos eletrônicos.

Como esta pesquisa se propôs a investigar o processo de desenvolvimento adotado, sendo este um evento contemporâneo e que está fora do controle do pesquisador, pode-se afirmar que se trata de uma pesquisa de campo por meio de um estudo de caso que está alinhado e é representativo acerca do problema específico levantado.

Foi investigada, para esta pesquisa, uma empresa de desenvolvimento de hardware, tendo seu nome omitido por questões de confidencialidade. Quanto ao instrumento de coleta, tem-se a observação do ciclo de desenvolvimento de um projeto de produto executados pela empresa durante um período de 6 meses. Foram utilizadas também informações cedidas pela empresa (imagens, esquemáticos) e entrevistas com os diretores a respeito da decisão pela adoção da prototipagem rápida, a fim de analisar o processo de desenvolvimento e as soluções propostas.

\section{Estudo de Caso: Anpalises e Resultados}

A seguir serão apresentados os resultados do trabalho, ou seja, os diagnósticos e discussões do estudo de caso realizado na empresa que atua como laboratório de pesquisa e desenvolvimento de protótipos para novos produtos.

\subsection{Caracterização das atividades da Empresa}

A empresa estudada, situada na cidade de Fortaleza - Ceará, é caracterizada como uma microempresa, que atua como laboratório de pesquisa e desenvolvimento de protótipos para novos produtos, fazendo uso de tecnologias em hardware (dispositivos eletrônicos) e software (sistemas embarcados e aplicações). O desenvolvimento do protótipo pode partir de uma solução original ou uma solução existente melhorada ou adaptada para as necessidades e a realidade dos clientes.

Como modelo de negócio e atividade, a empresa participa de projetos de Pesquisa, Desenvolvimento e Inovação (PD\&I) financiados por órgãos do governo e/ou de fomento para a geração de novos negócios, empresas privadas que querem inovar seus processos ou criar novos produtos, ou ainda projetos internos, com financiamento próprio. Entre alguns produtos desenvolvidos pela empresa estão rastreadores de veículos e cargas, set-top box com acesso a TV Digital e aplicações de interatividade para consumidores (projeto financiado pela Agência Nacional de Energia Elétrica ANEEL), equipamentos de mídia para "digital signage" e gestão à vista, de baixo custo, e dispositivos aplicados à área de saúde.

O financiamento para a construção de um protótipo parte do princípio da importância e necessidade de testar e validar novos conceitos e possibilidades, analisar o uso, operação e viabilidade, assim como identificar problemas e limitações técnicas antes de serem realizados maiores investimentos com a produção e lançamento do produto no mercado. Caso o protótipo atenda os objetivos e requisitos, provando sua validade e viabilidade, é realizado então um novo investimento, por parte do cliente, na construção de um produto que será produzido com escala de mercado em 
indústrias especializadas e com a composição de novas camadas não consideradas na fase de protótipo, tais como homologação ou registro de patente, embalagens e manuais para o usuário final.

\subsection{Processo de Pesquisa e Desenvolvimento}

Desenvolver um protótipo para um novo produto, na empresa investigada neste estudo, envolve um processo que pode ter variações em função do tipo de serviço, produto ou tecnologia, aspecto este que fica mais evidente em dispositivos eletrônicos com alto grau de inovação. Apesar das variações no processo e adaptações ao produto desenvolvido, o trabalho tem início com uma análise da ideia do produto e seus requisitos, abordagens tecnológicas, mercado, prazos e custos, passando pela pesquisa técnica e desenvolvimento propriamente dito, e se encerra com a entrega do protótipo mínimo viável e funcional, em um lote pequeno, juntamente com as especificações técnicas e informações para testes do clientes e posterior fabricação do novo produto. De modo mais detalhado, temos as seguintes etapas:

1) Análise da ideia, levantamento dos requisitos e documentação das especificações;

2) Setup do Projeto, com gestão de etapas, cronograma, atividades, riscos e custos;

3) Desenvolvimento do esquemático do circuito;

4) Layout da placa de circuito impresso (PCB);

5) Modelagem do case e componentes mecânicos;

6) Simulação e testes do layout (via CAD);

7) Contato com fornecedores, aquisição de componentes e produção do PCB;

8) Desenvolvimento do firmware;

9) Desenvolvimento do software embarcado e interfaces (dependendo do projeto);

10) Impressão em $3 D$ dos cases e componentes mecânicos;

11) Montagem do Protótipo;

12) Testes mecânicos, eletrônicos e de funcionamento do firmware/software;

13) Ajustes finais;

14) Elaboração de relatórios técnicos e especificações;

15) Entrega ao cliente.

Algumas etapas são executadas em paralelo, como o exemplo do desenvolvimento do software, que é realizado por outra equipe técnica especializada diferente do time do hardware, considerando também o fato de que não há dependência entre essas duas camadas até que o hardware esteja pronto. Por outro lado, algumas etapas dependem de espera externa, relacionada com a compra de componentes eletrônicos e a produção terceirizada da placa de circuito impresso (PCB), muitas vezes negociados com fornecedores nos EUA e China.

A multidisciplinaridade da equipe é outro aspecto marcante da empresa, tendo em vista que a concepção de um produto envolve diversos planos: eletrônica, mecânica, qualidade, design, firmware e software, além das competências relacionadas de engenharia de produto, inovação, gestão de projetos e aquisições.

Apesar da importância de todo o processo para a criação do protótipo, e cada uma delas oferecer seu próprio conjunto de problemas e soluções, é evidenciado neste estudo e foco de análise, especificamente, as etapas relacionadas a produção do dispositivo eletrônico (hardware).

\subsection{Desafios na producãa dos prototipos de hardware}

O desenvolvimento de novos produtos, e especialmente envolvendo alta tecnologia (hardware e software), oferece desafios que merecem a atenção da empresa desenvolvedora, dos clientes e dos profissionais envolvidos no projeto. Alguns desafios são comuns às empresas que fazem pesquisa e desenvolvimento, a exemplo do alinhamento de expectativas e necessidades dos clientes, o levantamento e análise de requisitos, a comunicação geral e o feedback durante o desenvolvimento, e a gestão do projeto como um todo, tratando dos prazos, custos, atividades, equipe e riscos. 
Contudo, alguns desafios são inerentes a produção de hardware, sendo este o foco desta investigação. Considerando o processo de desenvolvimento adotado pela empresa, comentado no item 4.2 deste artigo, tem-se alguns pontos específicos da produção de hardware, e do contexto de atuação da empresa, que oferecem dificuldades, de acordo com observações e relatos dos diretores e técnicos:

- Desenvolvimento do esquemático do circuito e layout do PCB: essas etapas convertem as especificações do produto em um circuito eletrônico, projetado e organizado em uma placa de circuito impresso (PCB). Para este trabalho, deve-se respeitar padrões e um conjunto de boas práticas, através do dimensionamento dos componentes e proteções, estratégia de elaboração do circuito visando seu melhor desempenho, a posição dos conectores de entradas e saídas de sinais e alimentação, a interferência entre regiões e a isolação, compatibilidade e posicionamento dos componentes do circuito, trilhas e camadas da placa. $\mathrm{O}$ desenho do circuito em CAD (Desenho Assistido por Computador) é realizado, de forma estruturada, levando-se em consideração o conhecimento técnico dos especialistas (GUERATTO, 2014). Ao final dessa fase, é gerada a lista de componentes e as informações de referência, quantidade, descrição, fabricante e número de série dos elementos, que serão usados para o desenvolvimento e montagem do dispositivo. A maior dificuldade neste processo é o design do circuito e da placa, que deve ser projetado de forma a atender as especificações em um menor tempo, gerando um circuito com o menor tamanho, consumo e custo possíveis, equilibrando com a complexidade para montagem, estrutura do laboratório, equipe técnica, terceirizações e aquisição de componentes.

- Aquisição de componentes eletrônicos: a partir do design do circuito e placa, com a definição de como o circuito vai funcionar, é gerada uma lista de componentes (Bill of Material - BOM) para aquisição. A problemática nesta etapa se relaciona ao custo, prazo e disponibilidade. No Brasil há ainda uma grande dependência de outros países - principalmente EUA, Japão, Israel e China - no que se refere a aquisição de componentes eletrônicos, tendo em vista que o país não tem um parque industrial especializado e capacitado para a produção da maioria desses componentes, aliado as patentes de uso. Essa dependência gera como complicadores os custos de importação (câmbio, frete e impostos) e os prazos para recebimento. Outro fator é uma limitação de disponibilidade de alguns componentes para aquisição, questão essa relacionada aos acordos comerciais entre os países. No caso dos EUA, por exemplo, alguns componentes não são comercializados para o Brasil, ou são necessários documentos que justifiquem o uso dos componentes para que os EUA autorizem a venda. Essa dificuldade em especial pode ser contornada no projeto do circuito, com a escolha de outros componentes similares para as funções especificadas e que estejam disponíveis, porém implicando em uma maior demora nessa fase de projeto.

- Necessidade de terceirização na produção do PCB: a produção da placa de circuito impresso (PCB) corresponde a uma etapa que deve ser terceirizada, por se tratar de uma construção complexa realizada apenas por indústrias especializadas. Dependendo da complexidade do PCB, e de forma similar a aquisição de componentes citada anteriormente, é necessário contratar empresas de outros países - principalmente China - em função das limitações do parque industrial brasileiro. Neste ponto, o aspecto mais crítico é o prazo, tanto pela duração do processo de produção e transporte do PCB, quanto pelo caráter de importância para o projeto, já que diversas etapas da produção do dispositivo dependem do PCB produzido, disponível e testado.

- Tempo de desenvolvimento, tempo de mercado e custos: problemática apontada pela empresa tem um caráter mais complexo e está ligada ao tempo do desenvolvimento e seus custos. Os custos para desenvolvimento de protótipos, no contexto nacional, são elevados e impactam nos projetos principalmente para micro e pequenas empresas, como o caso da empresa estudada neste artigo. Como exemplo o impacto do câmbio, taxas, transporte, impostos de importação e custos na aquisição e produção de componentes do projeto. A gestão do tempo do desenvolvimento, neste contexto, torna-se crucial no sentido de que 
atrasos, retrabalho ou projetos de longa duração implicam em altos custos. O fator tempo também está relacionado ao "time to market", ou tempo de mercado, aspecto um pouco mais subjetivo, mas igualmente importante para a empresa e seus clientes. Devido aos avanços tecnológicos e alta competitividade entre as empresas, é um fator estratégico para todo o negócio lançar um produto o mais rápido possível. $\mathrm{O}$ atraso no lançamento de um produto pode fazer com que este perca mercado ou nem mesmo consiga a viabilidade para ser lançado. Considerando o tempo necessário para pesquisa e criação do protótipo, o processo de desenvolvimento não pode suportar problemas como atrasos, retrabalho ou esperas longas por fornecedores, implicando em um prazo curto nessa fase de protótipo.

\subsection{Ferramentas e soluções adotadas pela empresa}

Devido, principalmente, aos prazos e os custos inerentes a realização dos Projetos, a empresa estudada optou por fazer investimentos no sentido de melhorar seu processo de design e prototipagem, entendidos como críticos para o desenvolvimento.

O primeiro ponto foi a aquisição e adoção de uma ferramenta CAD (computer-aided design) especialmente para eletrônica, no desenho dos circuitos (esquemático) e layout da placa de circuito impresso (PCB), o Altium Designer. A suíte de ferramentas permite, além do design, também a aplicação de simulações, testes e consegue gerar automaticamente a BOM (bill of materials), inclusive com comunicação online com alguns fornecedores de componentes eletrônicos. A figura 3 apresenta o esquema do circuito desenvolvido pela empresa.

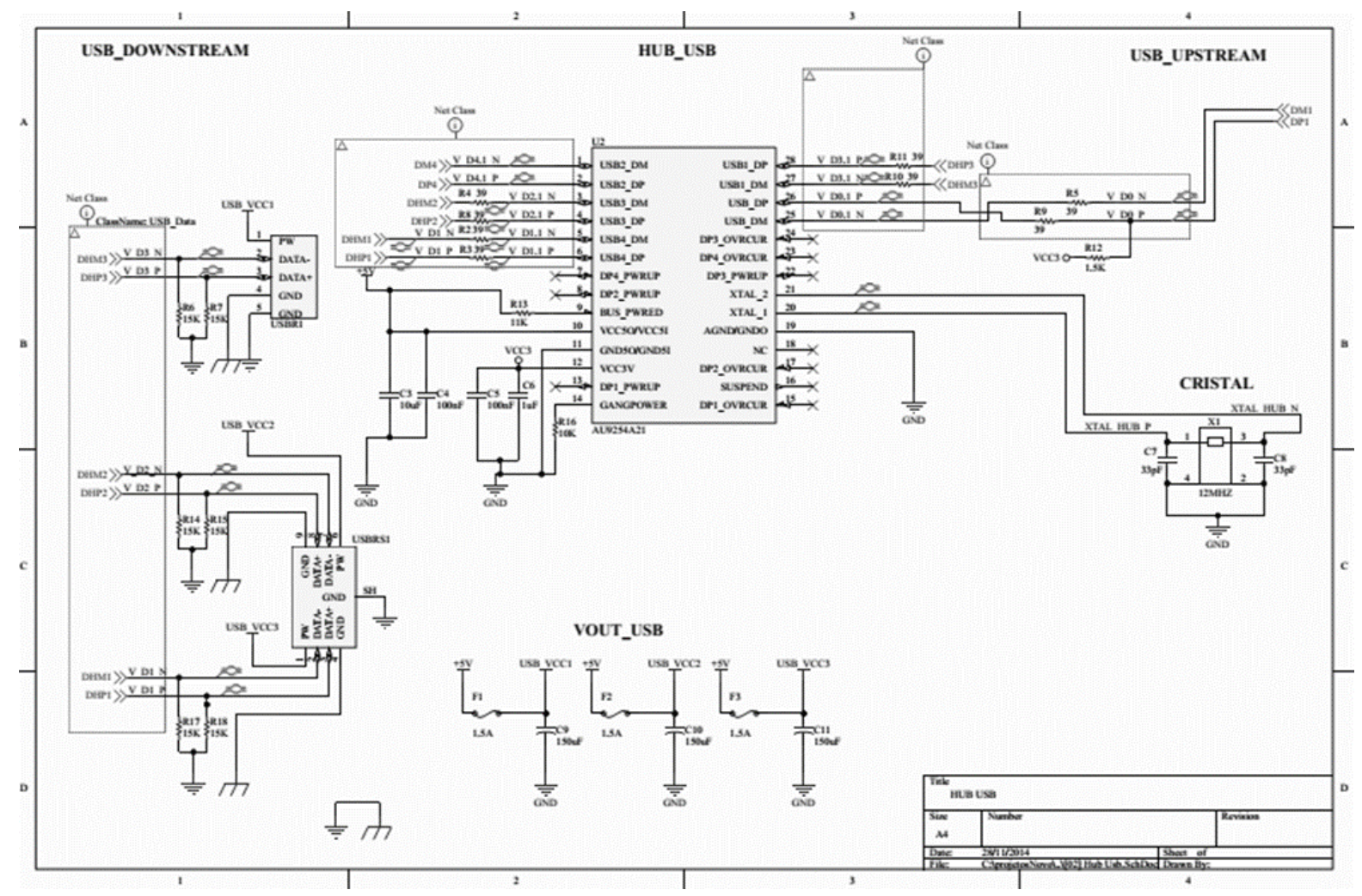

Figura 3 - Esquema dos circuito desenvuelto pela empresa

Fonte: Empresa pesquisada

Como exemplo, é apresentado o uso das ferramentas de prototipagem, especializadas no design de componentes e dispositivos eletrônicos, no processo de desenvolvimento de um set-top box, um dispositivo de mídia que pode ser conectado a TV convencional, funcionando como receptor do 
sinal de TV Digital e media center. A figura 4 apresenta o Design 3D do PCB desenvolvido pela empresa.

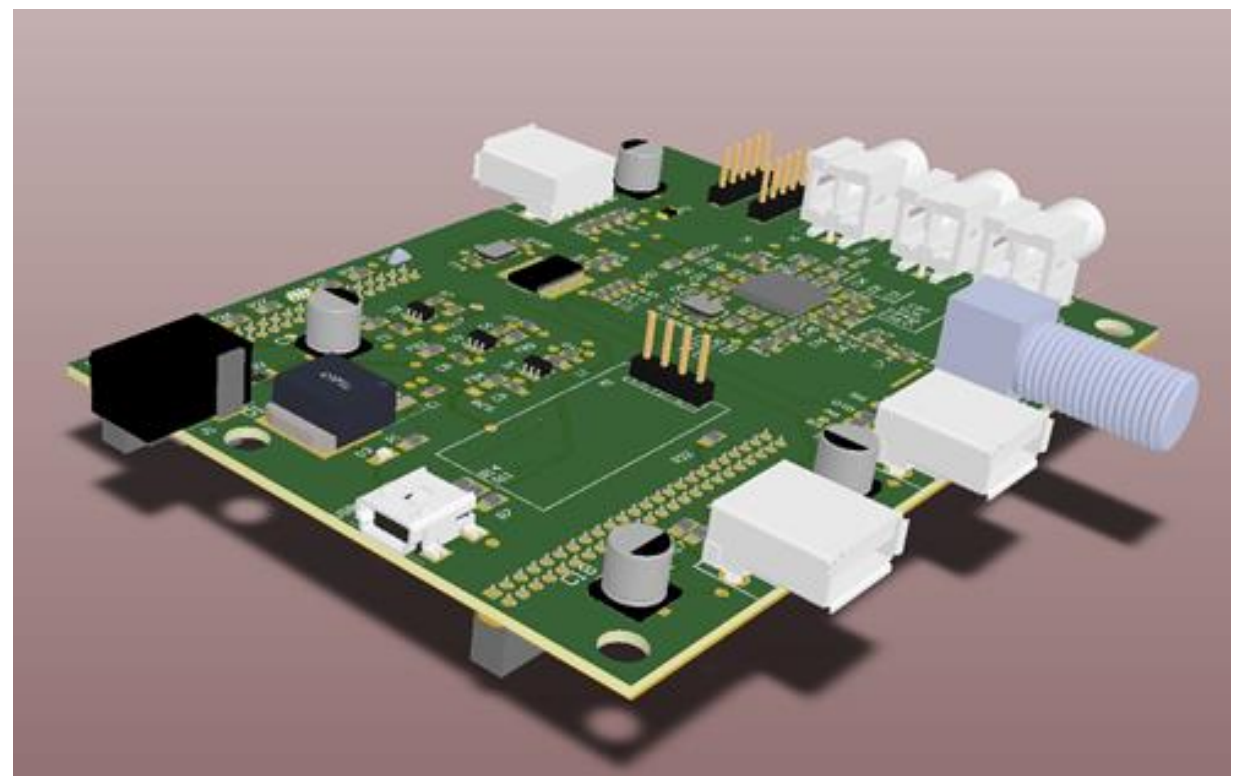

Figura 4 -Design 3D do PCB desenvuelto pela empresa Fonte: Empresa pesquisada.

A figura 5 apresenta o Design do PCB desenvolvido pela empresa.

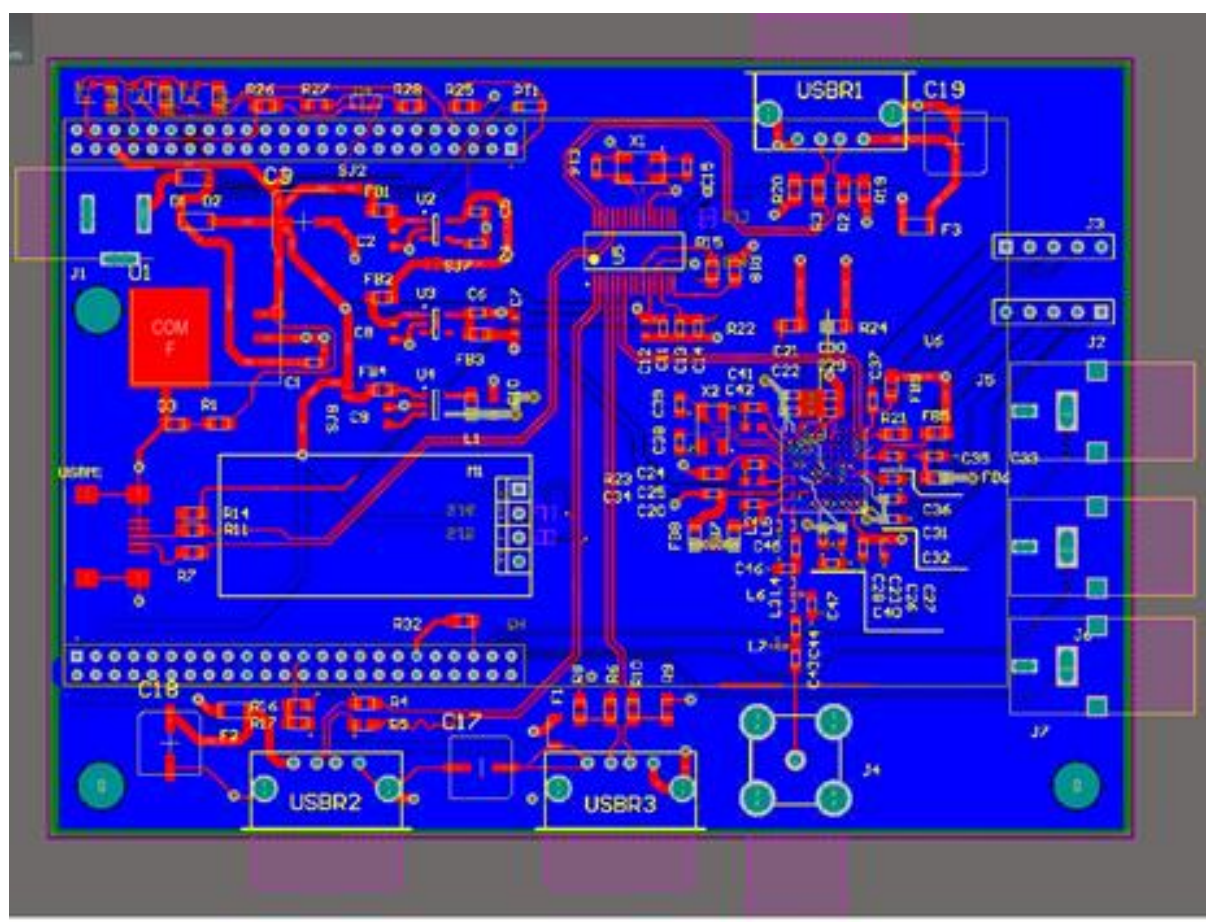

Figura 5 - Design do PCB desenvolvido pela empresa Fonte: Empresa pesquisada.

Por fim, a figura 6 apresenta uma placa montada pela empresa. 


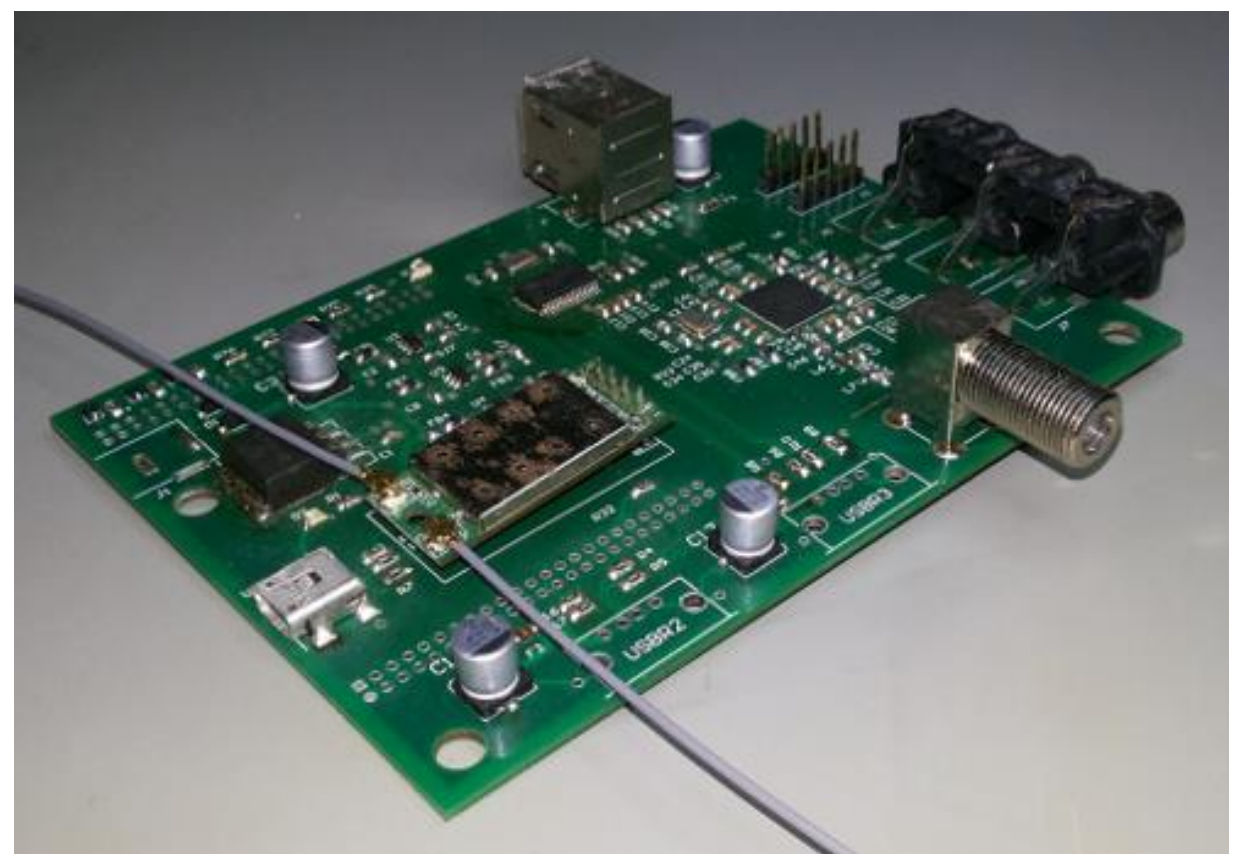

Figura 6 - Placa montada pela empresa

Fonte: Empresa pesquisada

A figura 7 apresenta um exemplo da BOM, ou lista de materiais, que é gerada automaticamente pelo software de CAD/CAM como saída do design do dispositivo.

\begin{tabular}{|c|c|c|c|c|c|c|}
\hline Digi-Key Part Number & Mandlacturer & Manufacturer Part Nun & Description & tity 2 & & \\
\hline 455-2247-ND & JST SALES AMERICA INC & $\mathrm{B} 2 \mathrm{~B}-\mathrm{XH}-\mathrm{A}(\mathrm{LF})(\mathrm{SN})$ & CONN HEADER XH TOP $2 \mathrm{~h}$ & 50 & 0,0856 & 4,28 \\
\hline AE9926-ND & ASSMANN WSW COMPONENTS & $A U-Y 1008-R$ & CONN USB RTANG FMALE & 50 & 0,8004 & 40,02 \\
\hline PJRAN1X1U02X-ND & SWITCHCRAFT INC & PJRAN1X1U02X & CONN JACK IPOS R/A PH, & 50 & 0.6284 & 31,42 \\
\hline A1923-ND & TE CONNECTIVITY AMP & $640456-6$ & CONN HEADER VERT $6 P O$ & 50 & 0.2084 & 10,42 \\
\hline WM4202-ND & MOLEXINC & 0022232041 & 1 CONN HEADER 4POS .100, & 50 & 0,258 & 12,9 \\
\hline 587-1785-1-ND & TAIYO YUDEN (VA) & JMK107BJ475KA-T & CAP CER 4.7 UF $6.3 \mathrm{~V} 10 \% \times$ & 100 & 0,0428 & 4,28 \\
\hline P39.0CCT-ND & PANASONIC ELECTRONIC COMP & ERJ-6ENF39ROV & RES SMD 39 OHM $1 \% 1 / 8 \mathrm{~W}$ & 700 & 0,012 & 8,4 \\
\hline 1276-1295-1-ND & SAMSUNG ELECTRO-MECHANIC & CL10C101JC8NNNC & CAP CER 100PF $100 \mathrm{~V} 5 \% \mathrm{~N}$ & 150 & 0,0237 & 3,555 \\
\hline P75.0CCT-ND & PANASONIC ELECTRONIC COMP & ERJ-6ENF75ROV & RES SMD 75 OHM $1 \% 1 / 8 \mathrm{~W}$ & 50 & 0,0288 & 1,44 \\
\hline 712-1297-1-ND & JOHANSON TECHNOLOGY INC ( & .500R07S330GV4T & CAP CER $33 P F 50 V 2 \% \mathrm{NPV}$ & 150 & 0,14 & 21 \\
\hline $445-4881-1-N D$ & TDK CORPORATION (NA) & $\mathrm{C} 1005 \mathrm{COG} 1 \mathrm{H} 050 \mathrm{CO5}$ & CAP CER 5PF 50V COG 046 & 100 & 0,0121 & 1,21 \\
\hline P43.2LCT-ND & PANASONIC ELECTRONIC & ERJ-2RKF 43R2X & RES SMD $43.2 \mathrm{OHM} 1 \% 1 / 1$ & 50 & 0,0196 & 0,98 \\
\hline $311-1361-1-N D$ & YAGEO (VA) & CCO805ZRY5V9BB $10 *$ & CAP CER 0.1UF 5OV Y5V OS & 300 & 0,01176 & 3,528 \\
\hline P14851CT-ND & PANASONIC ELECTRONIC COMP & ELJ-QF5N1DF & FIXED IND 5.1NH 350MA 1 , & 100 & 0,217 & 21,7 \\
\hline P10.0KCCT-ND & PANASONIC ELECTRONIC COMP & ERJ-6ENF $1002 \mathrm{~V}$ & RES SMD $10 \mathrm{~K}$ OHM $1 \% 1 / 8$ & 150 & 0,0219 & 3,285 \\
\hline $785-1159-1-N D$ & ALPHA \& OMEGA SEMICONDUC7 & $A O Z 1212 \mathrm{Al}$ & IC REG BUCK ADJ $3 A$ ASO, & 50 & 1,3448 & 67,24 \\
\hline CTX833CT-ND & CTS-FREQUENCY CONTROLS ( $\mathrm{V}$ & 405C 11A $12 \mathrm{M} 00000$ & CRYSTAL 12MHZ 1OPF SMb & 100 & 0,9 & 90 \\
\hline P11.OKCCT-ND & PANASONIC ELECTRONIC COMP & ERJ-6ENF $1102 \mathrm{~V}$ & RES SMD 11K OHM $1 \% 1 / 8$ & 50 & 0,0288 & 1,44 \\
\hline P12.4KLCT-ND & PANASONIC ELECTRONIC COMP & ERJ-2RKF $1242 X$ & RES SMD $12.4 \mathrm{~K}$ OHM $1 \% 1$, & 50 & 0,0196 & 0,98 \\
\hline P12233SCT-ND & PANASONIC ELECTRONIC COMP & EVQ-PC105K & SWITCH TACTILE SPST-NG & so & 0,2424 & 12,12 \\
\hline 609-1010-1-ND & $\mathrm{FCl}(\mathrm{VA})$ & 10029449-001RLF & CONN RCPT HDMI TYPE A & 50 & 1,018 & 50,9 \\
\hline P15.0KCCT-ND & PANASONIC EI & ERJ-6ENF $1502 \mathrm{~V}$ & RES SMD $15 K$ OHM $1 \% 1 / 8$ & 500 & 0,012 & \\
\hline PCD1925CT-ND & PANASONIC ELECTRONIC COMP & ELJ-RF1N5DFB & FIXED IND 1.5NH 400MA 7 , & 50 & 0,0698 & 3,49 \\
\hline B540C-FDICT-ND & DIODES INCORPORATED (VA) & $B 540 \mathrm{C}-13-\mathrm{F}$ & DIODE SCHOTTKY 4OV 5A, & 50 & 0,7656 & 38,28 \\
\hline P20.0KCCT-ND & PANASONIC ELECTRONIC CON & ERJ-6ENF2002V & RES SMD $20 K$ OHM $1 \% 1 / 8$ & 100 & 0,0219 & 2,19 \\
\hline
\end{tabular}

Figura 7 - Exemplo da BOM (bill of materials).

Fonte: Empresa pesquisada.

Além da ferramenta de CAD, a empresa adquiriu uma impressora 3D para a construção dos cases (gabinetes) dos protótipos. A impressora 3D entra, neste processo, como um elemento fundamental de Prototipagem Rápida, através do qual, apesar do alto investimento inicial na compra da impressora 3D, foi possível criar os cases e testar o design mais adequado, inclusive em conformidade com a placa de circuito projetada.

Foi escolhida a impressora 3D Makerbot Replicator 2, de pequeno porte, que utiliza como elemento para impressão o PLA (ácido polilático ou poliéster termoplástico), comumente derivado de recursos renováveis, como o amido de milho, raízes de mandioca ou de cana, biodegradável e nãotóxico. A figura 8 mostra a Impressora 3D Makerbot Replicator 2. 


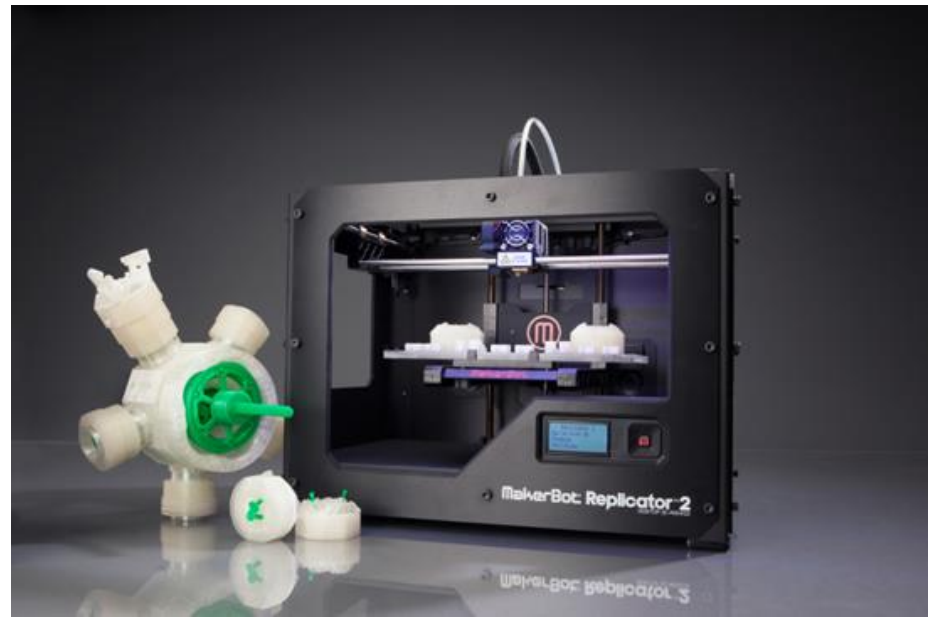

Figura 8 - Impressora 3D makerbot Replicator 2

Fonte: Empresa Pesquisada

A figura 9 apresenta um exemplo de gabinete impresso em 3D.

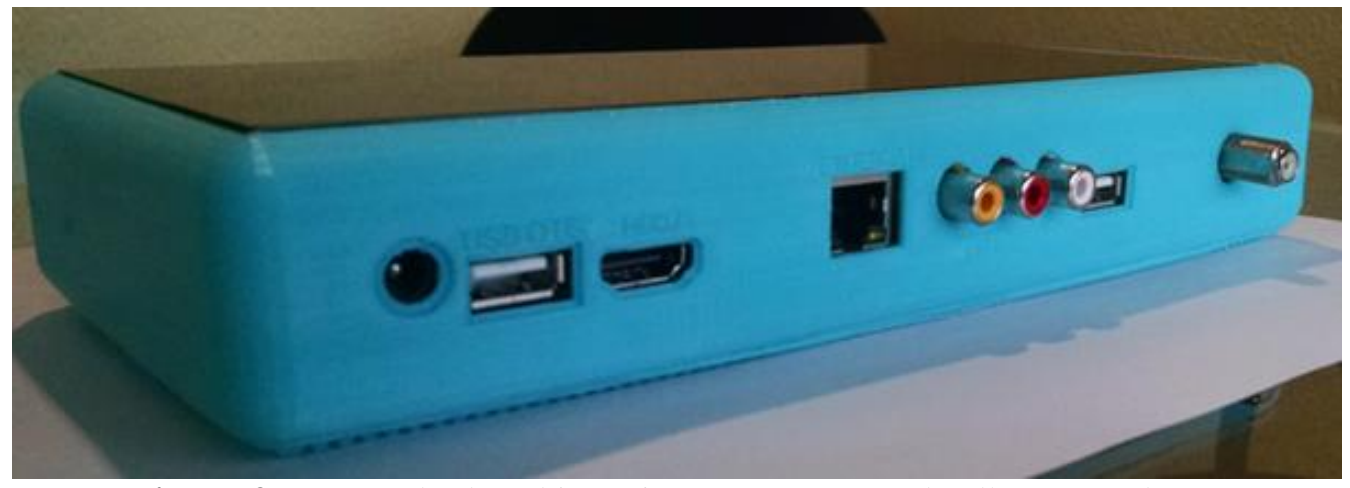

Figura 9 - Exemplo de gabinete impresso em 3D (detalhe em azul).

Fonte: Empresa pesquisada.

A figura 10 apresenta o design do case (gabinete com a placa).

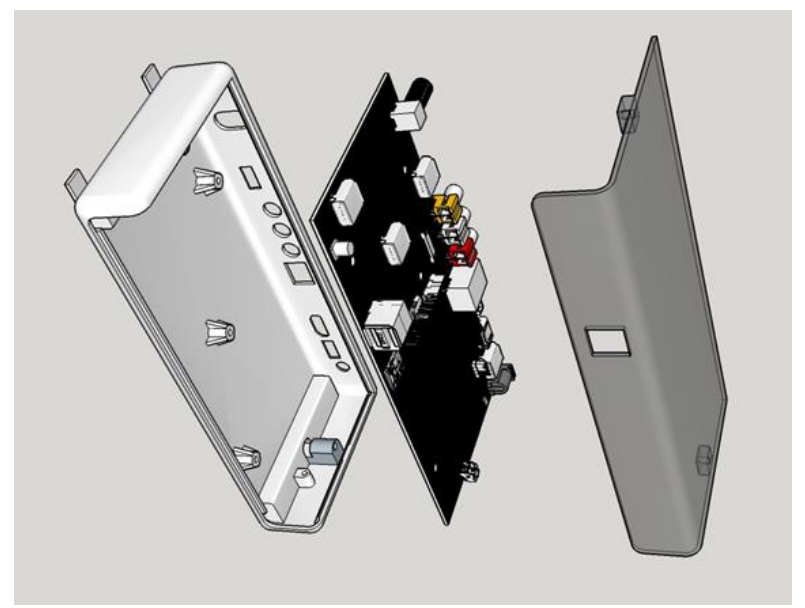

Figura 10 - Design do case (gabinete com a placa) Fonte: Empresa pesquisada 
Os resultados do investimento e adoção das ferramentas de CAD e impressão 3D para a empresa estudada, conforme relatos e observações, são apresentados a seguir.

\subsubsection{Redução de custos na produção dos gabinetes plásticos e ganho de tempo}

Apesar do investimento inicial relativamente alto para a aquisição do software e impressora 3D, houve benefício para a empresa com a redução de custos na fabricação dos gabinetes plásticos do protótipo. Antes da impressão 3D, a empresa contratava um fornecedor para a produção dos gabinetes, com um custo maior devido a pequena escala para protótipo (viável apenas para grandes lotes), e com uma espera maior, em torno de 2 semanas. A impressão 3D de um gabinete plástico (figura 9) pode ser concluída em 3 horas (devido a limitações da impressora 3D e de sua tecnologia), mas ainda assim mais rápido, prático e flexível em detrimento a terceirização. No caso observado, em um dia foi possível imprimir e testar 2 modelos diferentes de gabinetes plásticos para o protótipo. Outro importante impacto é a redução do time do market, garantido uma maior versatilidade e rapidez na construção do protótipo. O cliente, usuário do produto, também teve acesso ao protótipo em um curto período de tempo, gerando feedback para a melhoria do produto.

\subsubsection{Melhoria na comunicação, planejamento e qualidade}

Um ou mais modelos impressos em 3D, prontos e disponíveis em questão de horas, facilitou consideravelmente as atividades de planejamento, solução de problemas, projeto, comunicação e brainstorming, tendo em vista que é mais intuitivo e prático para a equipe discutir, medir, propor ideias e identificar falhas com uma representação física do produto ao invés de um desenho técnico ou descrição verbal, e em uma fase ainda anterior a produção. Como consequência deste aspecto, tem-se a redução dos riscos do projeto e a garantia de maior qualidade do produto final.

\subsubsection{Competitividade}

O investimento realizado com a impressora 3D e software CAD garantiu um maior destaque da empresa no mercado, comparativamente a outras empresas do mesmo segmento, além da imagem de maior confiabilidade e segurança para os clientes a partir da percepção destes em relação ao uso, por parte da empresa, de novas tecnologias como diferencial e na promoção da qualidade. Segundo relato dos diretores da empresa estudada, foi possível conquistar novos clientes a partir do momento que a empresa apresentou uma estrutura ágil de desenvolvimento de protótipos e validação de idéias, sem os altos custos e tempo de espera com a dependência da terceirização na produção de cases e modelos.

\subsubsection{Aquisição de Componentes}

O uso das ferramentas de CAD e, no geral, com a maior facilidade de gerar o protótipo impresso em 3D, teve como consequência uma melhoria no processo de compra de componentes. Não apenas pelo software gerar de forma automática a lista de materiais para a compra, mas pela praticidade na atualização ou mudança de componentes por questões de preço ou indisponibilidade no fornecedor. $\mathrm{Na}$ fase de design, com a ferramenta CAD, é possível rapidamente, e em tempo real, consultar a disponibilidade e o preço do componente eletrônico inserido no projeto da placa, diretamente com o fornecedor. No caso de indisponibilidade, rapidamente pode ser colocado outro componente no projeto. No caso do uso das duas ferramentas em conjunto, era possível ajustar o gabinete plástico em função da placa de circuito, como também, com a percepção de alguma inconsistência no modelo impresso, era possível fazer ajustes no design da placa, novamente 
atualizando a lista de materiais para a aquisição. Esse fluxo e a conexão entre as partes agilizou o processo como um todo, e a compra de componentes foi mais assertiva.

\section{Conclusões}

De acordo com as informações levantadas neste estudo de caso, as observações e depoimentos obtidos, conclui-se que o uso da prototipagem rápida, com a adoção da impressão 3D e softwares para prototipagem, impactou positivamente a empresa, desde redução de desperdícios, tempo, melhoria nos processos e maior agilidade e qualidade para os protótipos desenvolvidos.

Os resultados são ainda mais expressivos levando-se em consideração o contexto onde a empresa atua e seu porte, dispondo de limitados recursos para produzir protótipos e manter o alto nível tecnológico e de qualidade.

No caso da impressão 3D, conseguir produzir um protótipo a partir de um desenho eletrônico sem envolver outros processos ou terceiros aumenta as chances de detectar falhas de projetos nas fases iniciais, onde ainda não houveram grandes investimentos, aspecto que foi decisivo para a empresa na concepção de seus protótipos com várias mudanças durante o projeto e um prazo curto de execução. Mesmo com algumas dificuldades da impressão 3D, como o alto custo da impressora e sua manutenção, e os detalhes do acabamento do protótipo plástico, ainda assim constituiu vantagem para a empresa estudada pelo fato de que, em um intervalo de algumas horas, é possível que os engenheiros tenham à disposição um modelo pronto para ajustes, testes, e facilitando um brainstorming de melhorias com a peça nas mãos.

Apesar de várias camadas da empresa ainda precisarem de melhorias, assim como um refinamento das próprias soluções aplicadas, fundamentalmente percebe-se a criação de uma cultura da melhoria, com o pensamento da busca por novas ferramentas de trabalho, a exemplo da impressão $3 \mathrm{D}$, demonstrando um aspecto positivo da assertividade no uso de recursos e, consequentemente, na capacidade de se manter relevante e competitiva no mercado.

Este estudo demonstra ainda, de forma geral, as melhorias e as possibilidades, sobretudo para o desenvolvimento de protótipos, proporcionadas pelo crescente avanço das tecnologias no cenário da Prototipagem Rápida.

\section{Referências}

BARBOSA FILHO, A. N. Projeto e Desenvolvimento de Produtos. São Paulo. Atlas 2009.

BROWN, Tim. Design thinking. Harvard Business Review, v. 86, n. 6, p. 85-92, jun. 2008.

BROWN, Tim. Uma metodologia poderosa para decretar o fim das velhas ideias design thinking. Rio de Janeiro: Elsevier, 2010, 249 p.

ELVERUM, Christer W., WELO, Torgeir. On the use of directional and incremental prototyping in the

development of high novelty products: Two case studies in the automotive industry. Journal of Engineering and Technology Management, 2015, pg. 71-88.

GERHARDT, T. E.; SILVEIRA, D. T. Métodos de pesquisa. Porto Alegre: Editora da UFRGS, 2009.

GUERATTO, D. H. et al. Aplicação da Espiral do Conhecimento no Processo de Serviços ou Produtos de uma empresa de desenvolvimento de hardware. XXXIV Encontro Nacional de Engenharia de Produção, Curitiba, 2014. 
GORNI, A. A. Introdução à prototipagem rápida e seus processos. Revista Plástico Industrial. São Paulo, p. 230-239, 2001.

KAMINSKI, Paulo Carlos, DA SILVA, Guilherme Canuto. Selection of virtual and physical prototypes in the product development process. The International Journal of Advanced Manufacturing Technology, 2015.

MODEEN, T. CADCAMing: the use of rapid prototyping for the conceptualization and fabrication of architecture. Automation in construction, 2005. v.14, 215-224.

PINHO, A. F.; LEAL, F. \& ALMEIDA, D. A. A Integração entre o Mapeamento de Processo e o Mapeamento de Falhas: dois casos de aplicação no setor elétrico. In: Anais do XXVI ENEGEP, Fortaleza - CE, 2006.

ROZENFELD, H. et al. Gestão de desenvolvimento de produtos: uma referência para a melhoria do processo. São Paulo: Saraiva, 2006. 542 p.

SANCHES, V. R. et al. O Projeto Informacional do Desenvolvimento de uma Impressora 3D. XXXV Encontro Nacional de Engenharia de Produção, Fortaleza 2015.

TAKAHASHI, S. \& TAKAHASHI, V. P. Gestão de inovação de produtos: estratégia, processo, organização e conhecimento. Rio de Janeiro: Editora Campus, 2007.

VOLPATO, Neri. Prototipagem Rápida: tecnologias e aplicações. São Paulo: Editora Blücher, 2007.

YIN, R. K. Estudo de Caso: Planejamento e Métodos. 5.ed. Porto Alegre: Bookman, 2015. 